\title{
THE PLANT AND THE MANAGEMENT OF THE PICC / MIDLINE. THE PRIORITY NURSING ROLE IN THE APPROPRIATE CARE
}

\author{
${ }^{1}$ Cimmino Olimpia, ${ }^{2} \mathrm{D}$ 'Auria Raffaella, ${ }^{2}$ Sanselmo Salvatore, ${ }^{2}$ Castaniero Luisa, ${ }^{3}$ Falconio Lucio Marcello \\ ${ }^{1}$ Vaccination HUB Manager and regional reference for SDO flow ASL NA 1 Centro \\ ${ }^{2}$ Nurse ASL NAPOLI 1 CENTRO \\ ${ }^{3}$ executive pharmacist ASL NAPOLI 1 Centro
}

KEYWORDS: PICC management, appropriate care, nursing,

\section{ABSTRACT}

The interaction between the individual and the environment has a strategic significance for welfare purposes and shows the importance of the bilateral nature of the relationship. It highlights how promoting health means acting on the environment and on the individual.

In terms of health education, for health workers it is no longer a question of intervening from the outside on behavior, but of inserting themselves into situations, in the processes of interaction, as active protagonists of a complex system. This orientation of the health system requires a reinterpretation of the role of operators, through the adoption of the participatory planning method, which requires interpreting one's work as a problem solving process. To get to the solution of the problems it is necessary to identify the elements of difficulty that the patient encounters, thus adopting methodologies that allow for an exchange relationship. Nurses, in all health systems, have a privileged position due to the capillarity of their action, inherent in the "to care" of nursing care, and of their contacts with citizens and clients. In particular towards the patient he teaches the correct assistance maneuvers and the most appropriate attitudes to be adopted to favor the change of behaviors and the achievement of awareness of a new state of health and a new degree of autonomy.

The most suitable educational method that should be used by the nurse in the path of therapeutic education, is to link learning to action, in clinical practice, alongside the patient, promoting healthy lifestyles and the dissemination of a culture of health. Educational intervention aims at personalizing care and can improve health status and reduce healthcare costs.

Alongside the ethical value and professional duty, it is necessary to use methodologies, techniques and methods to provide a structured set of specific information, with a simple and understandable language, speaking.

\section{INTRODUCTION}

Slowly, carefully and in a suitable place, checking the end of the

communication the degree of understanding reached by the patient.

The educational project for patients waiting for PICC (Peripherally Inserted Central Catheter) or MIDLINE implantation was conceived with two main purposes :

1. Effectively inform patients waiting for PICC and MIDLINE implantation on the management of the device or on possible complications related to its use;

2. Promote continuity of care and thus contribute to improving the patient's state of health and quality of life .

The scientific evidence affirms that, today, the number of patients who refer to oncological, haematological treatments or in any case to infusion therapies continued for long periods is increasing sharply.

The lack of adequate venous access leads to: delayed initiation of the parenteral therapeutic and nutritional plan; lengthening of hospitalization days; increased phlebitic, thrombotic complications; extension of nursing times to find access, increase in patient discomfort and stress.

The use of the P.ICC (Peripherally Inserted Central Catheter) and the MIDLINE has seen a significant increase, being considered a safe method for the long- term administration of antibiotics, chemotherapy, parenteral nutrition, both in hospital and for home care. , as well as usually well tolerated by the patient and easy to insert. The PICC can be inserted by trained nurses inclusion, recognition and management of complications, as well as the medication and the follow up of patients.

\section{Anatomical Aspects}

In this chapter is explicit anatomy in reference to veins, arteries and nerve supply of the upper limbs. The knowledge of anatomy is of fundamental importance for the location of the aids to be treated in the thesis topic. Knowledge of the anatomical location is important to avoid the onset of complications such as an accidental arterial puncture or risk of injury to the nerves affecting the limb.

\section{Arteries and veins}

The channels that carry the blood form a closed system. In the arteries, blood circulates to the periphery, in the veins to the heart. The capillaries are thin small vessels interposed between arteries and veins, where the exchanges between blood and tissues take place. The wall of the vessels is made up of three layers, or cassocks, superimposed: the intimate cassock, the innermost and thinnest, the medium cassock, the thickest, and the adventitious cassock . In addition to the common constructive plan, arteries and 
veins were formed by the same tissue components: connective with predominance of elastic fibers, muscle tissue smooth with fibrocells to disposal circular or a spiral, and endothelium.

Arteries generally have thicker walls than veins, as they have to withstand significantly higher blood pressure. They have a whitish color, are rather consistent and elastic. Even if empty of blood they retain the open light. The intimate cassock is formed by an endothelial lining that rests on an elastic lamina .

The medium tunic, always quite thick, is made up of connective tissue rich in elastic fibers and collagen fibers. Smooth muscle tissue is always present, in varying quantities depending on the type of arteries. In some it predominates over the elastic component. Finally, the adventitia is made up of an intertwining of fibrous connective tissue, plus or less often, which has the function of preventing the vessel from becoming worn out.

Arteries can be divided into:

1. Arteries of the elastic type (diameter greater than 5-6 mm)

2. Muscular type arteries (less than $5 \mathrm{~mm}$ in diameter)

The main characteristic of the elastic-type arteries (aortic trunk, common carotids, subclavian vessels, common iliacs) is to optimally oppose the pressure thrust of the circulatory stream. The district arteries are of the muscular type, that is, those that supply sufficiently delimitable portions or districts of the body (intestine, lower limb). The renal arteries, which are of the elastic type, and those of the head and upper limb are exceptions. Finally, the smaller caliber arterioles are always of the muscular type .

The elastic arteries pulsate in synchrony with the heart, that is, at each ventricular systole there is a pulsation of the artery, even if it follows with a certain interval with respect to the systole, an interval the longer the greater the distance of the vessel from the heart. It is known that if an artery is cut in vivo, the blood comes out intermittently: this phenomenon is in fact due to cardiac activity. The arteries have a containment function of the thrust of the blood and favor the propulsion, both through the active contraction of their own walls (muscular arteries), and an elastic reaction. For example, the main branches from the aorta directed to the limbs (of the elastic type) accumulate a lot of elastic energy following the thrust of the heart, and use it to progress the blood towards the periphery. Their structure allows to transform the intermittent flow of blood into a continuous flow.

The muscular type arteries, however, require contractile structures to compensate for the decrease in pressure (usually are far from the heart): the their contractility helps the path of the blood and regulates the flow to each organ. While the distribution territories of the large and medium caliber arteries are rather constant, individual variations of the territories served by the smaller arteries are frequent, and it is noted that the frequency of these variations increases going towards the periphery. Usually the spraying territories are served by several arterial branches connected to each other, but there are important districts sprayed by terminal arteries, vessels that lead individually to a territory, which is thus sprayed by a single artery. The pulmonary, splenic branches, cerebral and coronary arteries are of the terminal type .

The arteries tend to be located deep in the tissues, and to accompany two veins, called satellite veins; only rarely are they superficial, subcutaneous.

The veins, carrying the blood back to the heart, bear much lower blood pressure than the arteries. Originally they have a very small diameter and very thin walls, but as they approach the heart they merge with other veins, increase in size and simultaneously become more consistent, even if in general their walls remain less thick than those of the arteries, although sometimes being larger in diameter. As in the latter, the wall is made up of three cassocks: intimate, medium and adventitious. Generally the muscular and connective fibrous component prevails over the elastic one.

The veins are divided into:

1. Venule

2. It is contentive

3. Propulsive or muscular veins

The venules have an extremely thin and not very resistant wall, mainly formed by connective tissue rich in collagen that surrounds the endothelium. Smooth muscle cells are scarce.

The content veins also have a wall rich above all in collagen fibers, at the level of both the intima and the media, where circular or longitudinal muscle bundles may be present. The elastic component is scarce.

Supradiaphragmatic veins belong to this type, that is, of the head, neck and upper limb, in which blood flows at low pressure. In the veins of the head and neck the pressure is simply due to the force of gravity. Propulsive veins are more similar to arteries, as they have a rather thick wall. In addition to the endothelium, the intimate tunic has an elastic membrane, while on the average the muscle bundles are abundant. An elastic connective layer is still present in the adventitia. Propulsive veins are those visceral, superficial circles and limbs. Even in these veins the blood pressure is low, and they have the task of actively favoring, with the contraction of the walls, the ascent of the blood towards the heart.

Unlike arteries, many veins have internally valvular devices: membranous folds in the shape of a swallow's nest, called semilunar valves . These remain slumped against the wall of the vessel when the blood flows towards the heart, but spread out by protruding into the lumen of the vein when the blood, by force of gravity, tends to flow back towards the periphery. They are present in those vessels where the gravity pressure is considerable, for example in the veins of the lower limb. Instead, they are missing in the superior cava, portal vein and pulmonary veins .

There are more veins than arteries. Simplifying we distinguish two groups of veins:

1. Superficial veins, with subcutaneous course, clearly evident in some individuals. They have no correspondence with the arteries.

2. Deep veins or satellites: they run in the muscular lodges or between the viscera accompanying the arteries in the territory from which they drain the blood. Often each artery is accompanied by two satellite veins. Satellite veins are formed at the level of the venous plexuses, devices of widely anastomosed post-capillary venules.

Anastomoses between veins are much more numer- 


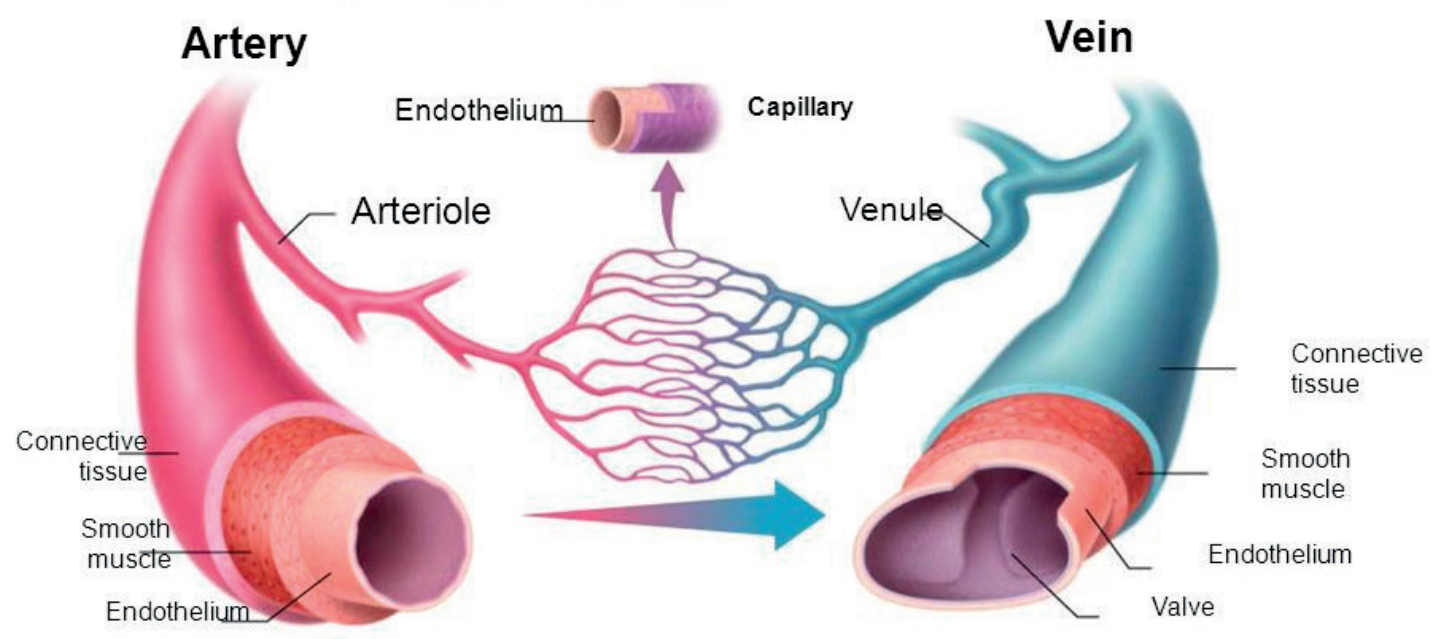

Fig.1 - arterial / venous vessel

ous than arterial ones. It is important to remember the anastomotic devices between the superficial and deep circulation, or those between the areas above and below the diaphragm. In addition to what has already been said about arterial anastomoses, it is worth remembering that veins, having very thin walls, can be compressed or occluded very easily. Therefore, collateral passages are necessary that equally allow the drainage of the isolated territory and the return of blood to the heart.

All the waste blood of the great circle reaches the heart through the two large hollow veins. The superior vena cava located in the right upper mediastinum is formed by the union of the two anonymous veins at the mouth of the thorax. The total path reaches about $7 \mathrm{~cm}$ while the diameter of the pot, when it is unfolded, can be greater than $2 \mathrm{~cm}$. It has an almost vertical course from top to bottom, with a slight concavity towards the left: while at its origin it is close to the sternum, at the sternoclavicular joint interline, descending downwards.

The inclination of the sternum progressively distances itself from the anterior thoracic wall, becoming a deep vessel: it can be projected on the anterior thoracic wall on a right sternal-marginal line, from the lower edge of the sternum manubrium, up to the second costal cartilage. Due to its relationships it is divided into two sections, one superior called extrapericardial, the other intrapericardial because it is wrapped in the pericardial sleeve of the veins.

The superior vena cava is a containing vein, with thin walls, mainly fibrous, it is not provided with valves: it does not receive other collaterals besides the azigos vein .

The anonymous veins, the trunks constituting the superior vena cava, are large, decidedly asymmetrical veins being the right vein much shorter than the left, which crosses the medial plane and moves to the right to merge with the contralateral vessel.

The two anonymous veins receive different tributaries which can however present considerable variations. The lower thyroid veins that carry blood reflux from the viscera of the neck constantly open both to the right and to the left: the internal mammary veins, the vertebral vein, the posterior jugular vein that collect parietal blood from the thorax and from the spine of the neck.

The subclavian vein represents the last segment of the waste venous bed of the upper limb: it has a considerable diameter of about $12 \mathrm{~mm}$ and a length of a few $\mathrm{cm}$. It begins as a direct continuation of the axillary vein and the limit is conventionally marked at the lateral margin of the first rib, it goes a little arch-shaped medially almost horizontal crossing the anterior face of the anterior scalene muscle at its section on the rib and thus reaches the point of confluence with the jugular vein. The subclavian vein receives the external jugular vein at its outlet: in a different way in the two antimers they open at the point of confluence with the anonymous, the large lymphatic trunks.

The internal jugular vein is a large vein that represents the large drainage vessel of the intracranial circulation and which during its path in the neck collects waste veins from the organs of the facial block and neck. It has an overall length of over $15 \mathrm{~cm}$ extending along the entire neck from the skull base to the point of confluence in the anonymous: it has an average diameter of about $12 \mathrm{~mm}$.

The inferior vena cava is formed by the confluence of the two common iliac veins, to the right of the bifurcation of the abdominal aorta. It rises, keeping to the right of the aorta, and collects along the way veins from the abdominal walls and viscera, and the important portal vein system.

\section{Anatomy of the arteries of the upper limb}

The arterial tree of the upper limb derives from a stem trunk continuation of the subclavian artery that first penetrates the stump and then extends undivided throughout the arm: in this section the arterial trunk, with the collaboration of some branches of the subclavian to the vascularization of the shoulder stump and of the two arm lodges, it is divided into two segments: the first which follows the subclavian contained in the axillary cavity, is called the axillary artery; the second tract located medially in the anterior lodge of the arm is the brachial artery. The latter extends anteriorly to the crease of the elbow where it divides into two main trunks that follow the two medial 
and lateral sides of the limb and are therefore called the ulnar and radial artery. Both, after having collaborated with the brachialis in the vascularization of the elbow, provide the structures contained in the anterior and posterior forearm loggia; arrive, then up to the wrist and penetrate the anastomosis hand with several arches and provide numerous branches, that will extend until the fingers. The complex is said arterial device of the hand and includes three arches, one dorsal and two palmares from which originate distally directed branches that follow the intermetacarpeal spaces and reach the fingers, vascularized with different digital arteries .

The subsequent arterial organs of the upper limb are located in the volar lodges in front of the articular joints: the branches that are carried dorsally are relatively small branches coming from the trunks located in the anterior lodges.

\section{Anatomy of the veins of the upper limb}

There is a deep and a superficial circle. The first faithfully follows the path of the arteries, originating at the level of the hand with arched digital veins that flow into two radial veins and two ulnar veins (satellites of the homonymous arteries). At the elbow they merge into the two brachial veins that finally make up the axillary vein, close to the homonymous artery. This continues with the subclavian vein, then with the anonymous vein that is thrown into the superior vena cava . The superficial venous system begins at the level of the hand with veins identifiable under both palmar and dorsal skin. The dorsal network gives rise to the basilica vein, which is a voluminous vein that represents the primitive superficial vein of the upper limb. Originating in the back of the hand, it is carried obliquely along the ulnar margin of the forearm but as it rises it becomes decidedly flying. After passing the joint line of the elbow, having received the median anastomotic vein, the basilica continues in the arm and approximately at the level of the lower III is placed in the medial bicipital groove: then it perforates the fascia and opens into the medial brachial vein.

The palmar network gives rise to the cephalic vein, with a lateral course, which anastomoses with the basilica at the bend of the elbow. Assai longest of the basilica originates at the level of the wrist and moves along the forearm medial margin then oblique anteriorly up to the crease of the elbow and beyond the joint line it is lateral to the biceps up to the antero-inferior margin of the deltoid muscle near the deltoid tuberosity. Here medially oblique placing in the gap between the deltoid and pectoralis muscle against the fascia and following that interval arrives just below the clavicle where perforates the clavi-pectoral fascia to debouch in the vein axillary shortly before of its passage in the vein subclavian.

There are some individual variations, for example the presence of a median vein may be found, a branch that the cephalic vein emits just before the bend of the elbow: directed obliquely upwards and medially reaches the basilica vein above the joint line and opens into it. When the vein is well developed, a characteristic venous device is shown on the volar side of the elbow formed on both sides by two major cephalic and basilica veins joined by the oblique anastomosis of the median vein. When the latter is underdeveloped there is a venous reticulum between its veins which is the continuation of veins located in the central volar area of the forearm and which at the crease of the elbow gives rise to veins that open into one or the other. other vein. They are called median veins of the forearm and present numerous variations from the case of an intermediate main vein between the cephalic vein and the basilica to the case of a network of veins without main elements.

Along the outer edge of the forearm may be the accessory cephalic vein, which reaches the cephalic just below the crease of the elbow.

\section{Upper limb innervation}

The brachial plexus is a voluminous formation that provides the skin and muscle innervation of the attachment stump and the free part of the upper limb, with the exception of the axillary area pertaining to the intercostal nerves.

The brachial plexus formations extend from the para-vertebral line of the cervical spine towards the shoulder stump and then into the free part of the limb. The different components are arranged as follows: roots, fascicles, nerves contained in muscle spaces or lodges of the base of the neck, of the shoulder stump and of the free part. The plexus is contained in the subclavicular and axillary spaces in a deep position, covered anteriorly by the conspicuous muscle bridges covering the neck and armpit: posteriorly, the coverage is even more impressive because it is represented by the entire scapular bone girdle with the respective muscles. The most superficial and accessible parts of the plexus are the two upper and lower extremes: the upper can be projected to the side of the rachis at the bottom of the supraclavicular fossa, the lower reaches the opening of the armpit and this part becomes decidedly superficial, if the limb is brought into abduction and elevation. In this case the nerve formations approach the skin covering plane of the armpit. We will then continue to analyze more specifically which are the nerves that are part of the upper limbs .

The radial nerve has a tortuous spiral path in the posterior lodge of the arm, from which it passes at the level of the elbow into the anterior lodge; when it reaches its terminal division it gives rise to the deep branch which passes into the posterior loggia where it runs up to the wrist. The superficial branch remains in the anterior lodge of the forearm, then passes into the back of the hand.

The median nerve originates in the axilla with two roots from the front two dossiers, and roots to comprise the eyelet axillary artery is then located on the front of the artery, medially with respect to the musculo-cutaneous nerve, laterally a little ' in ahead of to the ulnar nerve and to the two cutaneous nerves of the plexus. Overtaken the tendon of the subscapularis muscle and then the united tendons of the large round and the large backbone the nerve enters the arm and is placed in the anterior lodge at the front of the medial intermuscular septum in proximity and close relationship with the coraco-brachial muscle and the two homonymous veins in the nerve-vascular bundle of the arm.

The musculo-cutaneous nerve is a mixed nerve which has the characteristic of giving only muscle branches in the arm and only cutaneous branches in the forearm. Due to its overall characteristics it must be considered as a nerve belonging to the median nerve system, that is, a ventral nerve with a flexor value. It originates 


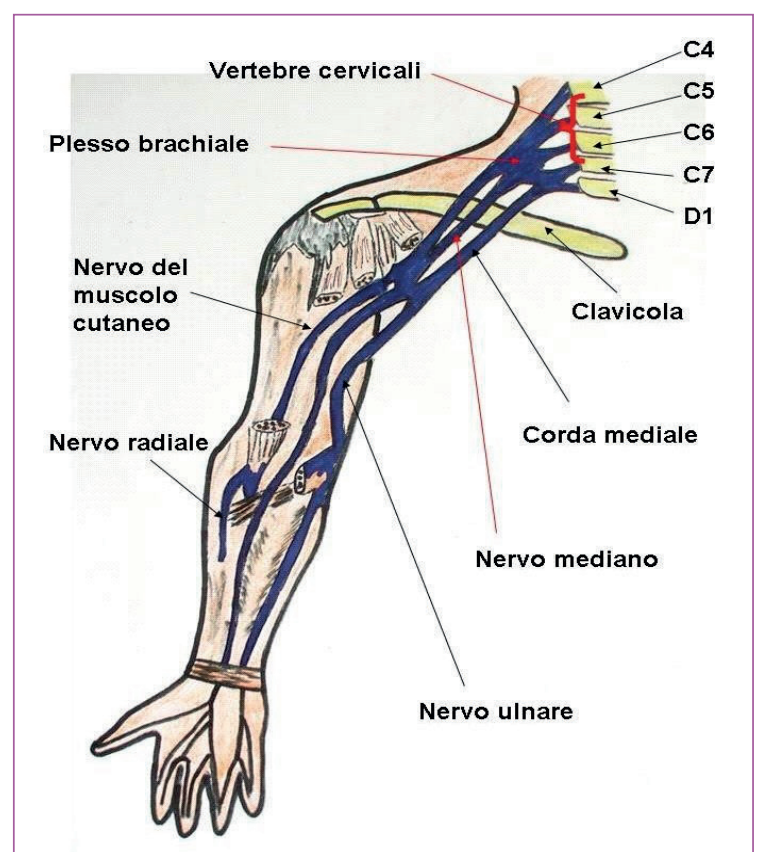

Fig. 2 - Upper limb innervation

in the armpit from the anterior-lateral fascicle together with the external median root: it is therefore the most lateral nerve of the plexus formation and is also deep resting directly on the subscapularis muscle. Both median and musculo-cutaneous are always volar, ie they are in the anterior space of the arm where the median becomes part of the vascular-nervous bundle; at the elbow it passes into the axis of the limb, and so into the forearm, then to the volar outer side of the hand.

The ulnar nerve is a mixed nerve that runs along the ulnar side of the limb; it supplies muscle branches to the forearm and hand, initially lies in the posterior arch of the arm, but then takes a volar course in the forearm and hand, except for its dorsal cutaneous branch. It originates from the armpit from the anteromedial fascicle together with the medial root of the median nerve and the two cutaneous nerves: at its origin it is the more medial nerve of the plexus and therefore relatively more superficial. After crossing the tendon of the subscapularis, dorsal and grand teres muscle, it settles behind and medial to the axillary artery. Entering the arm, the nerve moves a little away from the nerve-vascular bundle formations, placing itself in the posterior loggia, after having perforated the medial intermuscular septum .

The medial cutaneous nerve of the arm originates in the axillary cavity from the antero-medial fascicle and almost immediately becomes superficial, taking to the skin of the face external axillary cavity. It then descends vertically downwards, distributing cutaneous branches to the armpit, to the medial aspect of the arm up to the elbow: its skin area extends both anteriorly and posteriorly into the arm.

The medial cutaneous nerve of the forearm arises together with the previous one from the anteromedial fascicle of the plexus; it remains deeper at first, descending vertically just forward to the axillary artery and median nerve. It always remains below the fascia up to the lower third of the arm, where it becomes a satellite of the basilica vein; it then appears on the arm band becoming subcutaneous.

Venous Access And Its Appropriate Use

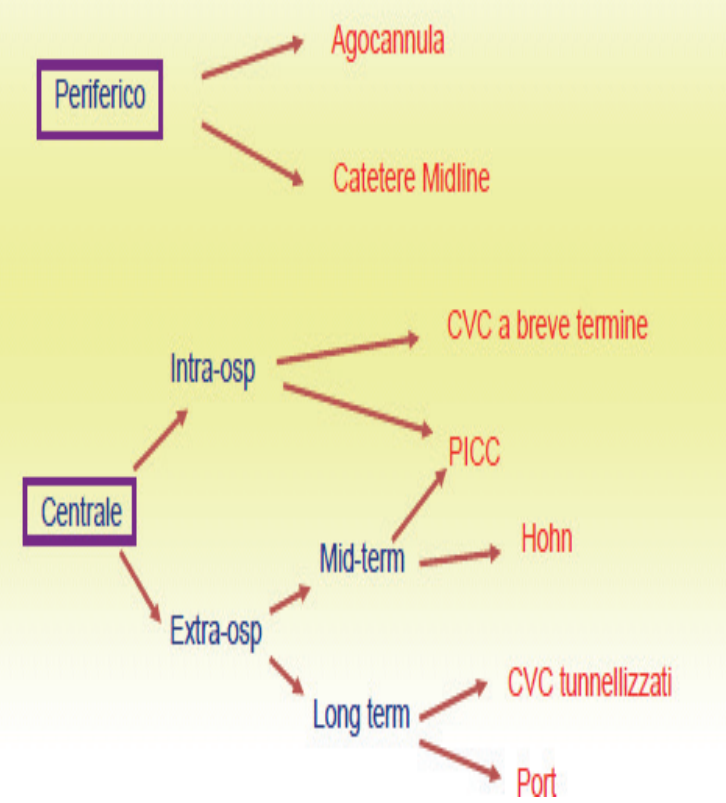

Fig. 3 - Algorithm for the choice of venous access in adults

\section{What is a venous access?}

By venous access we mean the introduction of a catheter or a needle into a vein, peripheral or central, previously selected by the doctor or nurse who will perform the procedure, which guarantees quick access to the venous stream for the infusion of liquids, nutritional solutions, drugs, blood and its derivatives.

Nurses have a fundamental role both in the management of venous access and in that of patient information and education. In particular, in the case of central venous access, the patient must be helped to accept the changes due both to the disease and to the presence of the venous device necessary to facilitate his treatment program, but certainly not without possible complications.

The training and continuous updating of nurses in this area are necessary to obtain uniform knowledge and behavior in the management of venous access and to prevent major complications, mechanical or infectious, linked to the presence of the system. In this way it will be possible to ensure the patient a "quality" nursing care that provides for patient satisfaction, his collaboration in the correct management of venous access, the absence of complications and the maintenance of the system for all the time required by the patient. treatment.

\section{What are the usable venous accesses? How to choose the right one?}

The venous accesses are divided into peripheral and central; the diagram below shows us schematically the specifications for each type of access based on their use and their field of application.

When it comes to choosing the most suitable venous access for the patient we are treating, we inevitably have to ask ourselves a series of questions:

- In what context do I need venous access (intra-hospital or extra-hospital)?

- How long will the venous access be needed (1-5 days, 1-4 weeks, months, etc.). 
The basilica vein

BENEFITS

- It is the vein of first choice for the insertion of PICC, it has larger dimensions than the cephalic;

- It follows a straight trajectory, the passage of the catheter into the axillary vein, into the subclavian, into the anonymous and therefore into the superior cavity, is easier

\section{DISADVANTAGES}

- It is not superficial like the cephalic, it is sometimes difficult to palpate

- It flows in the vicinity of the brachial artery and the medial branch of the cutaneous nerve of the forearm and therefore there is a risk of injury to these structures during venipuncture

- $\quad$ Do not use for patients who walk with the use of crutches

Tab. 1

\begin{tabular}{|c|c|c|c|}
\hline \multicolumn{4}{|c|}{ The brachial vein } \\
\hline & BENEFITS & & DISADVANTAGES \\
\hline (¿) & It is the second choice vein indicated & & Very close to the artery and nerve \\
\hline & when the veins superficial I'm & & brachialis \\
\hline & inaccessible & () & Risk of arterial puncture \\
\hline () & Pungible only under guide & & High venipuncture failure with \\
\hline & ultrasound & & blind technique \\
\hline
\end{tabular}

Tab. 2

\begin{tabular}{|c|c|c|}
\hline \multicolumn{3}{|c|}{ The cephalic vein } \\
\hline BENEFITS & & DISADVANTAGES \\
\hline () Third choice superficial vein & & During catheter insertion you can \\
\hline (¿) Used for obese patients and & & encounter resistence to it causes of the \\
\hline \multirow[t]{7}{*}{ edematous } & & presence of a more pronounced angle a \\
\hline & & level of the junction between vein \\
\hline & & axillary and subclavian and by presence \\
\hline & & of multiple venous valves \\
\hline & (2) & Tall risk from malposition for \\
\hline & & ascent in jugular internal or \\
\hline & & return back into the axillary vein \\
\hline
\end{tabular}

Tab. 3

- What kind of therapy should I give? (Blistering drugs, parenteral nutrition, hyper-osmolar, acidic, basic solutions etc.)

- I need of an access of type central or peripheral?

- What type of patient do I have in front of me (oncology, septic, hematology, dialysis, etc.)?

The veins that are used for the insertion of PICC/ MIDLINE type venous catheter inserted peripherally are:
Venous Accesses: Application Possibilities In Practice

PICC: description, advantages and disadvantages The PICC is a peripherally inserted non-tunneled central venous catheter (CVC): its tip is positioned near the junction between the superior vena cava and the right atrium; it can be in silicone or polyurethane, single or double-lumen. 


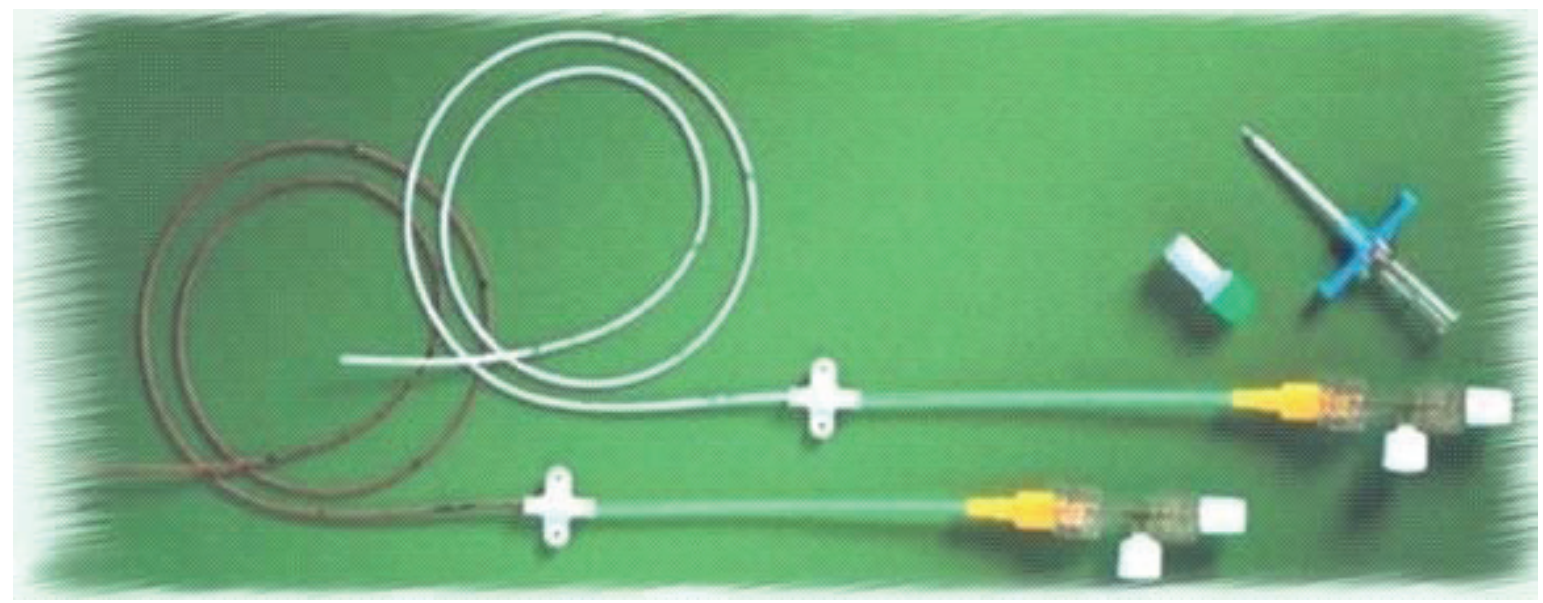

Fig. 4 - Central venous catheter with peripheral insertion

The PICC allows all the typical uses of "classic" CVCs: measurement of venous pressure, infusion of hypertonic solutions, administration of basic drugs $(\mathrm{pH}>9)$, acids $(\mathrm{pH}<5)$ or vesicants or irritants for blood vessel walls. It can therefore be used for nutritional treatments, chemotherapy and pharmacological therapies in which central venous administration is indicated.

The following situations are indications for choosing a central venous catheterization using PICC :

- Patients with high risk of complications mechanical if it proceeds to insertion of a CVC into an internal jugular or subclavian vein (obese patients; patients with anatomical and / or pathological changes in the neck; patients with severe coagulopathy);

- $\quad$ Patients with a high risk of infectious complications if a traditional CVC (patients with tracheostomy, immunosuppressed patients or subjects at high risk of bacteremia)

- Situations in which it is logistically difficult or expensive to proceed with the placement of a traditional CVC (domicile, lack of a dedicated team; etc.);

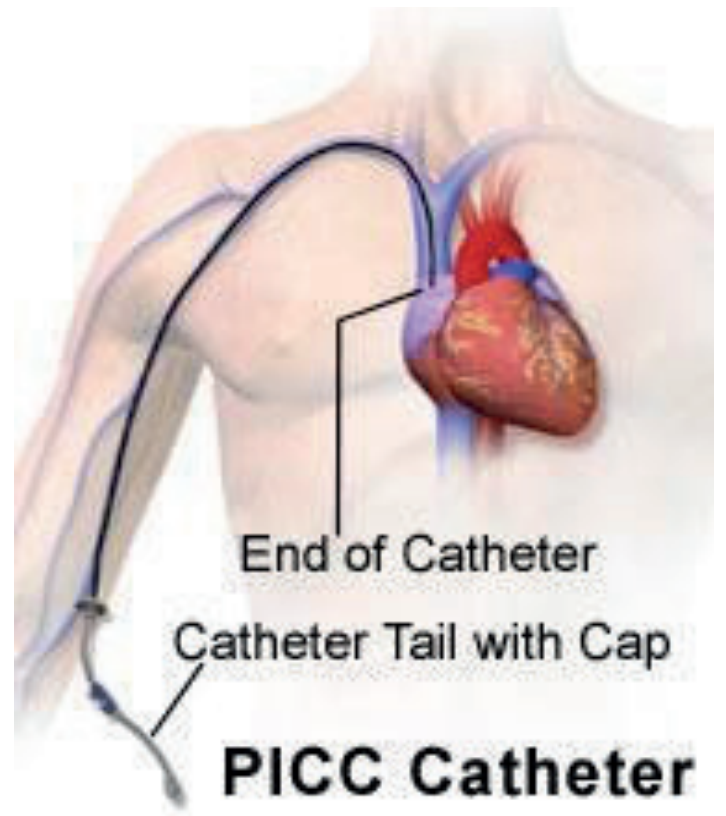

Fig. 5 - PICC positioning
- Need for central venous access for a particularly long time ("Medium term": $<3$ months);

- Medium-term need for central venous access $(<3$ months) in a patient to be treated also or exclusively in an out-of- hospital setting;

- Medium-term need for central venous access $(<3$ months) to be used also or exclusively in a discontinuous way .

The use of PICC as a CVC has the advantage of eliminating the risk of mechanical complications related to insertion, typical of direct central venipuncture (such as, for example, hemo-pneumothorax), of lowering the risk of bacteremic complications and of reducing the risk of central venous thrombosis; moreover, the positioning can be carried out by medical or nursing staff, adequately trained, and does not require anesthetic or surgical skills. This type of catheter can also be used discontinuously, without increasing obstructive or infectious complications, and can be used both intra and extra hospital.

However, in order to implant a PICC, both the practicability of the patient's peripheral venous heritage and the specific experience of the operators are necessary

in the implantation and management of the catheter itself.

PICC Features:

- Siliconeor Polyurethane

- $\quad$ Punta closed valved distal "Groshong" or tip open

- Equipped of Clamp of closure

- Length variable

- System of fastening with Sutureless Device that can be double or single

- Possible measuring PVC in PICC Power Polyurethane tip open

\section{Midline: Description, Advantages And Disadvan- tages}

The Midline (Figure 5.1), usually 20-30 cm long, although inserted like the PICC at the level of the arm or forearm using an ultrasound-guided technique, is not a central catheter because its tip is positioned at the level of the axillary vein or of the subclavian vein (Figure 5.2). It can be made of silicone or polyurethane, be single or double and can have a non-reflux valve (Midline Groshong). 


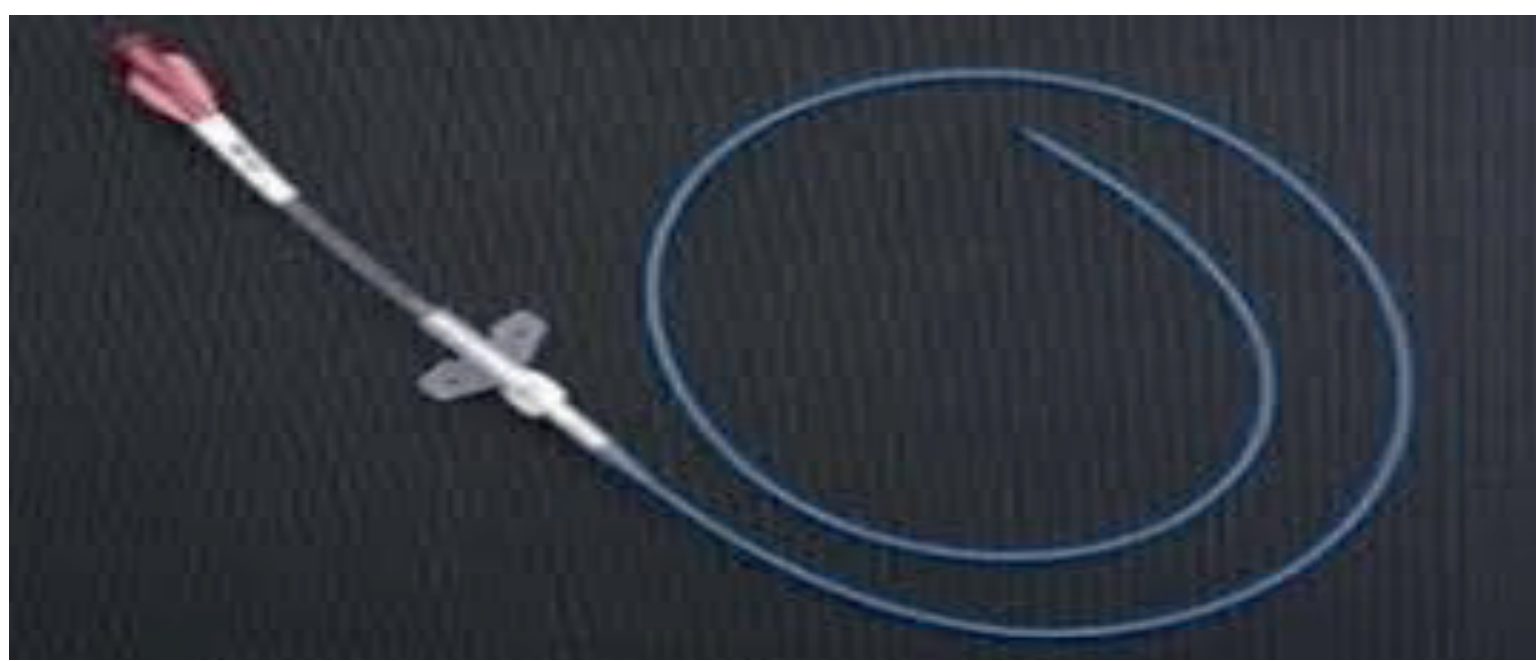

Fig. 6 - PICC Groshong (Silicone closed tip with valve)

The Midline, being a peripheral catheter, does not allow the typical uses of central venous catheters, it can therefore be used for pharmacological and nutritional therapies compatible with the peripheral pathway (osmolarity $<800 \mathrm{mOsm} / 1$, pH between 5 and 9, non-blistering drugs and not irritating to the endothelium).

The Midline can find indications for use in case of:

- Any treatment intravenous planned for more from 10 days, particularly in patients with poor peripheral venous stock ;

- Perioperative intravenous therapy in patients with poor peripheral venous heritage (e.g. obese, drug-dependent patients or patients suffering from

- $\quad$ chronic);

- Prolonged antibiotic therapies for severe infections (endocarditis, osteomyelitis, etc);

- "Life-saving" intravenous cardiological therapies for prolonged periods (cardiotonics, cardiokinetics, antiarrhythmics);

- Intravenous therapy in subjects with depleted peripheral veins, in which the central venous cath- eterization involves risks prohibitive in terms of immediate mechanical complications (respiratory failure, disorders of

- coagulation) or septic (immunosuppression and sepsis);

- Hydroelectrolytic or nutritional support for more than 10 days (neurological patients, complicated post-operative courses, acute pathologies conditioning the non-usability of the gastrointestinal tract until stabilization);

- Temporary venous access in patients awaiting definition of a definitive therapeutic program or undergoing removal of a garrison long-term venous for septic complications, and for which intravenous therapoy is indispensable ("bridge" catheter).

Compared to the average duration of a traditional venous cannula needle (3-4 days), the Midline catheter can remain in place for prolonged periods, even for weeks, with a very low risk of infectious or mechanical complications .

Further advantages are represented by the overcoming of the limit represented by the patient with depleted superficial venous patrimony, by the absence of

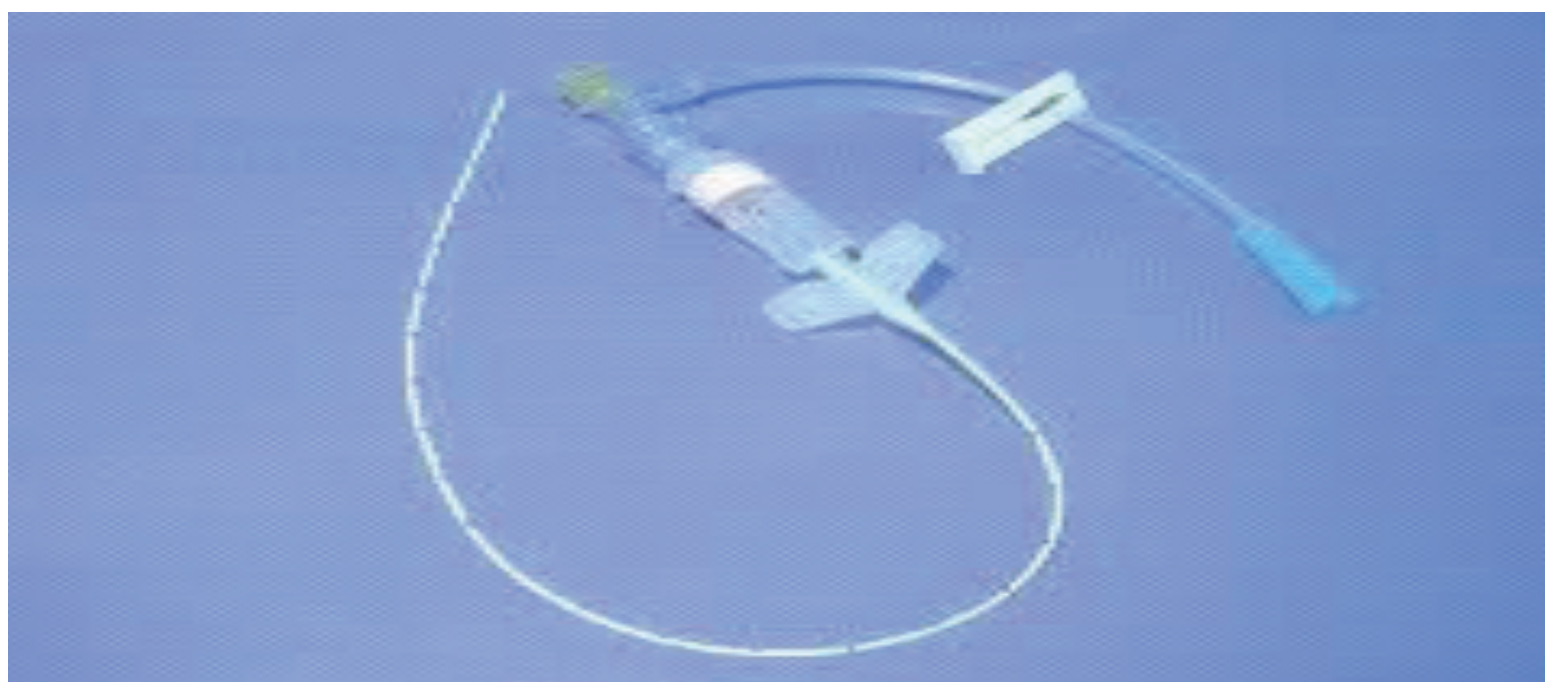



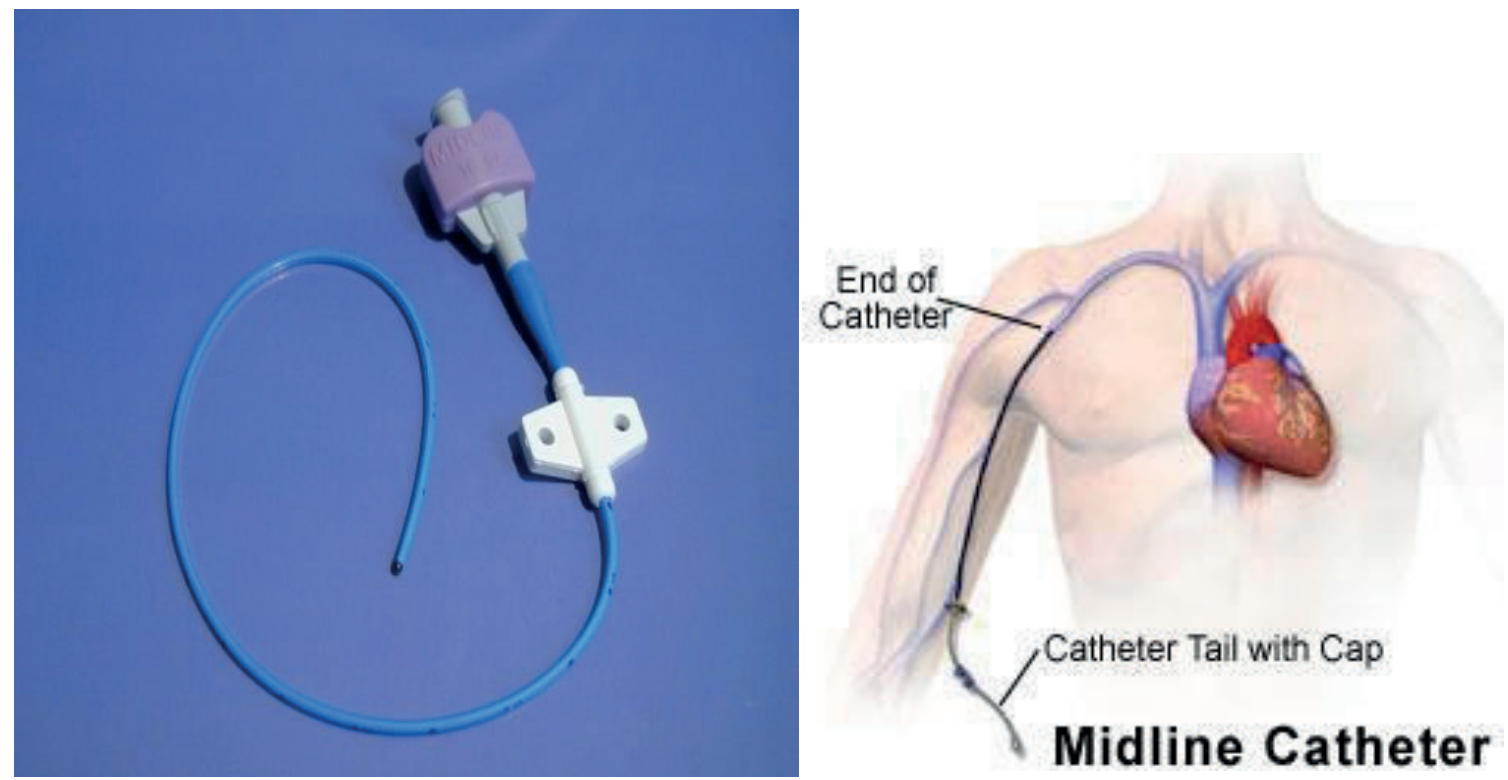

Fig. 8 - Midline positioning

major complications from bleeding in anticoagulated patients and by the fact that this nursing device is the device with the lowest rate of bacteremic infectious complications .

The disadvantages associated with the use of this catheter are represented by the need for specific implant know-how, by the possible local phlebitic and thrombo-phlebitic complications attributable to the ultrasound-guided implantation in the 3rd middle of the arm and limitation of movement and in the management of personal hygiene that the patient may encounter.

Characteristics of MIDLINE:

- Silicone or Polyurethane

- Punta closed valved distal " Groshong" or tip open

- Equipped with closing clamp
- System of fastening with Sutureless Device that can be double or single

\section{The Positioning Of A Venous Catheter And Its In-} dications

\section{Peripheral Insertion Central Venous Catheter (PICC) Insertion Procedure}

The decision on the type of system to be implanted is a collegial moment (doctor-nurse-patient if conscious).

It is a decision-making process in which the choices and assessments of the healthcare professionals must be shared with the patient and / or his family, taking into account the real objectivity.

For most oncological pathologies or therapeutic phases, the positioning of a venous catheter is foreseen by

\begin{tabular}{|c|c|}
\hline Human resources & $\begin{array}{l}\text { nurse implanter that needs to have received } \\
\text { specific training in the insertion of the PICC; }\end{array}$ \\
\hline Material Resources & 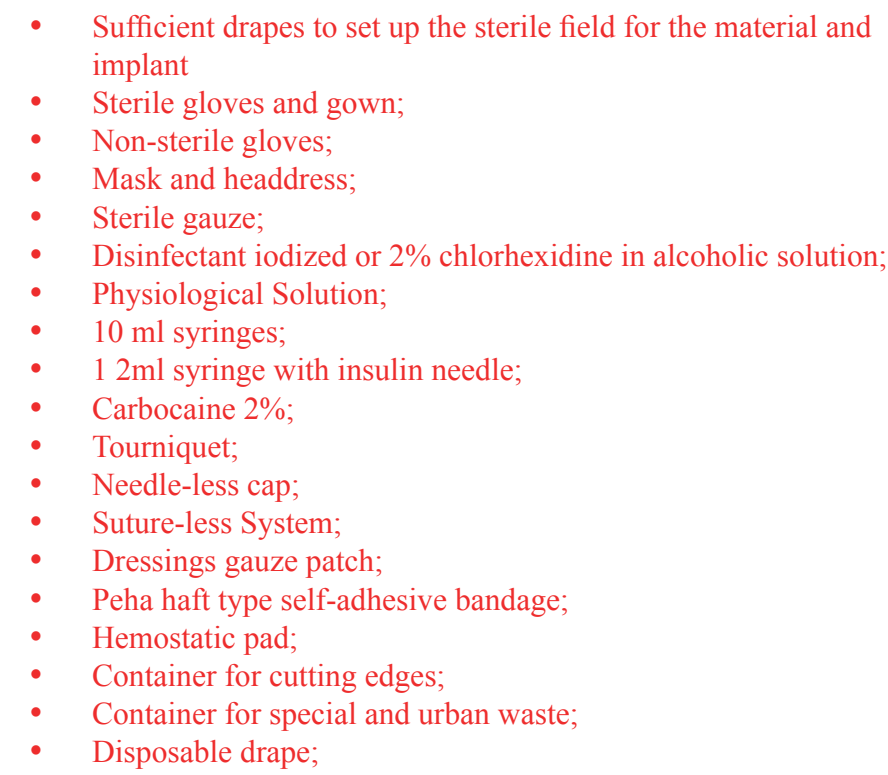 \\
\hline
\end{tabular}




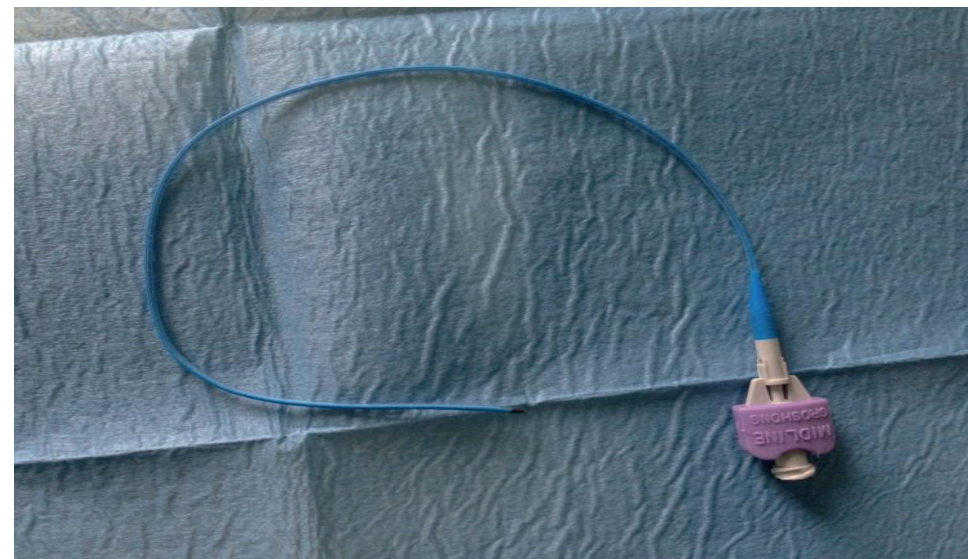

Fig. 9 - Midline Groshong (Silicone closed tip with valve)

the therapeutic protocol and is an indispensable aid for its correct application.

The venous catheter aims to ensure:

The stability of the venous access ;

- Avoid continuous venipuncture;

- Possibility of discontinuous use ;

- Duration guarantee for the period of treatment to be carried out;

- Maximum biocompatibility;

- The reduction of infectious and thrombotic complications ;

- Reduce the risk of accidental operator stings

Table 6.1 indicates the clinical situations in which it is advisable to evaluate the positioning of a CVC, in the medium or long term, both to preserve the patient's venous heritage and to reduce its discomfort by making therapeutic treatment easier and less invasive as possible .

\section{Peripheral Insertion Central Venous Catheter (PICC) Insertion Procedure}

Before choosing the vein in which the PICC is positioned, it is advisable to examine with the ultrasound system bilaterally all the deep veins of the arms (basilica, brachial) as well as the central veins of the supra-subclavicular region (axillary, subclavian, internal jugular, anonymous) to exclude any anatomical anomalies or previous venous thrombosis, and to be able to choose the vein most likely associated with a success of the maneuver.

For a long time, in fact, ultrasound was only seen as a way to obtain a safer and more precise venipuncture, underestimating the impact it can have in studying the veins before the procedure (this applies to both PICCs and traditional CVCs ) so that you can choose the most appropriate vein for the catheter

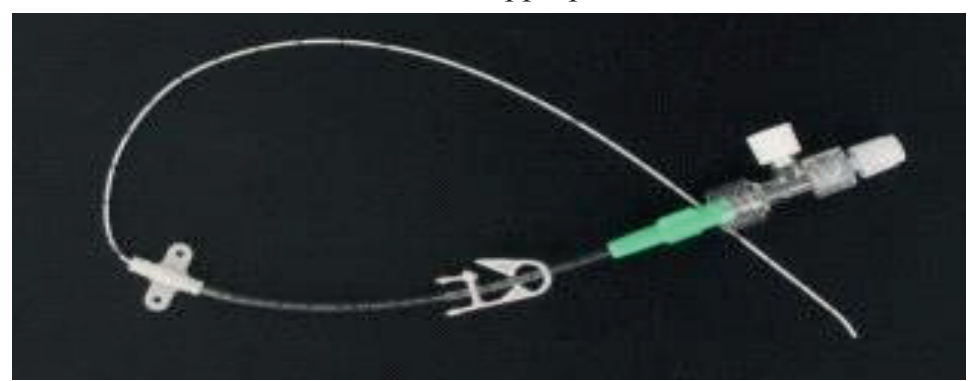

Fig. 11 - Midline open toe (Polyurethane)

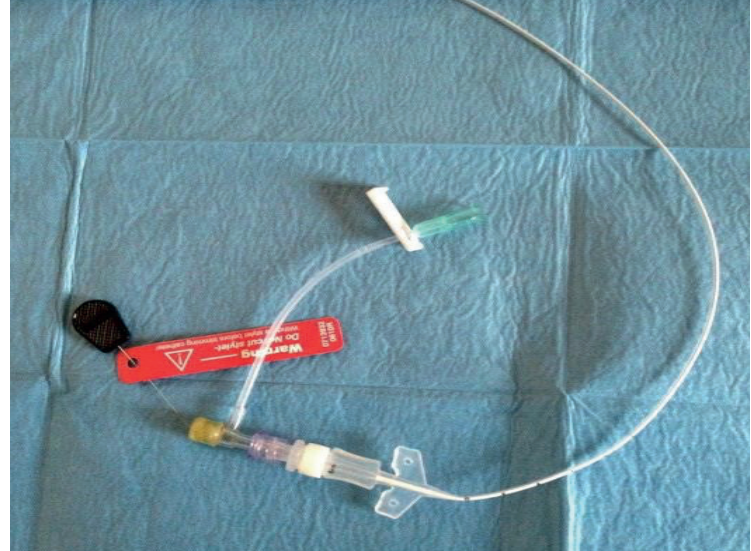

Fig. 10 - Midline open toe (Silicone)

in a given patient. Deep veins in the arm should be evaluated with and without a tourniquet. The choice of the optimal vein to cannulate is a crucial moment for the success of the maneuver and for the reduction of risks related to the procedure. The first important parameter to consider is the caliber of the vein: it is good to choose veins whose diameter, evaluated without tourniquet, is at least the triple of the catheter diameter (ie: the diameter in millimeters is equal to or greater than the catheter diameter French) thereby decreasing the risk of PICC-related peripheral venous thrombosis. Examples: $3 \mathrm{Fr}$ catheter: $9 \mathrm{Fr}(3 \mathrm{~mm})$ or larger vein ; $4 \mathrm{Fr}$ catheter : $12 \mathrm{Fr}(4 \mathrm{~mm})$ or larger vein ; $5 \mathrm{Fr}$ catheter : $15 \mathrm{Fr}(5 \mathrm{~mm})$ or larger vein ; 6 Fr catheter : 18 Fr $(6 \mathrm{~mm})$ or larger vein .

\section{Management of devices for venous access}

To ensure patient safety, the clinician (doctor or nurse) must be specifically competent in the management of venous access devices and must have knowledge of the anatomy, physiology and techniques of handling such devices in order to reduce the risk of complications.

Indications and protocols for the management of these devices must be specified in company procedures and / or local protocols, in compliance with the manufacturer's instructions. All components of the infusion line connected to the venous access device must be used only in the presence of a precise clinical indication, for a specific purpose and in accordance with the manufacturer's instructions.

Venous access devices must be stabilized and secured appropriately to reduce the risk of complications and / or the accidental loss of access. Methods used to stabilize the catheter should not impede evaluation and control of the emergency site and should not impede venous circulation or interfere with the delivery of infusion therapy. The venous catheters are to be stabilized and fixed using specific devices of stabilization designed for this purpose (engineered stabilization device, ESD).

Inadequate stabilization or fixation can result in accidental dislocation of the catheter with complications that often require early loss of venous access . In addition, the ESD facilitate the standardization of the same practices on the part of all clinical; reduce the complications secondary to the excessive mobility of the catheter in the emergency site; reduce the risk of forced interruptions of infusion therapy; help reduce 


\section{Echo - guided insertion procedure of PICC type catheter}

1) Explain the procedure to the patient in detail ;

2) Collect the patient's verbal and written consent to the procedure and check for any presence of pacemakers or implantable defibrillators (in this case the PICC will be placed in the contra-lateral limb) and that there are no known allergies ;

3) Rating ultrasound of the veins of the arms first without and then with the application of the tourniquet tourniquet;

4) Evaluate the theoretically accessible veins, identify the insertion point with a dermographic pen and remove the tourniquet;

5) Measure the length of the catheter to be introduced (distance from the insertion point to the midclavicular + distance to be hemiclavered at the 3rd right intercostal space );

6) Carry out social hand washing;

7) Place the patient in a supine position, with the arm at 90 degrees, palm of the hand up;

8) Perform trichotomy if necessary;

9) Place the disposable drape under the patient's arm ;

10) Wear a mask and headgear;

11) Perform antiseptic hand washing;

12) Put on the sterile gown and gloves;

13) Prepare the sterile field by opening the non-adhesive drape on the work surface;

14) Place the necessary material on the sterile field: Sterile drapes with adhesive

- 1 sterile drape without adhesive

- Ampoules of physiological solution; Syringes $10 \mathrm{ml}$;

- 1 from $2.5 \mathrm{ml}$ syringe with needle from insulin;

- $\quad$ Sterile gauze ;

- $\quad$ Tappino needleless / Port protectors; Two gauze dressings to patch; Carbocaine $2 \%$

- Hemostatic pad;

- $\quad$ Sutureless system;

- Patches steri-strip;

- Sterile probe cover;

- Microintroduction kit ;

- $\quad$ Pre- packaged PICC kit

15) Draw the 2 vials of physiological sol into the $10 \mathrm{ml}$ syringes

16) The second operator applies the tourniquet;

17) Sterile single-use and single-dose applicator of $2 \%$ chlorhexidine in $70 \%$ AIP

18) Respect the action time of the disinfectant;

19) Place 1 sterile drape without adhesive under the arm intended for implantation and apply 2 adhesive sheets on the arm, leaving the insertion site in evidence

20) Check dilator and peel-away and wash them with saline, extract metal guide ;

21) With there but no dominant, hold the introducer needle present in the kit;

22) With the non-dominant hand, hold the ultrasound probe, protected by the sterile probe cover, keeping the visualization of the chosen vein in the center of the screen to facilitate venipuncture;

23) Prick and advance with the needle at a 45 - 60 degree angle to the skin surface;

24) Gently insert the metal guide until the skin is about $20 \mathrm{~cm}$ outside ;

25) If the guide does not progress, try to extract it slowly without forcing, if this is not possible, first extract the needle and then the guide;

26) Remove the needle from the skin being careful not to remove the guide;

27) Remove the tourniquet;

28) Run wheal of Carbocaine to $2 \%$ in the seat of the emergence of the puncture guide;

29) Make a small skin incision $(2 \mathrm{~mm})$ with the scalpel in a horizontal position at the emergence of the guide;

30) Introduce, with a screwing movement, the preassembled dilator to the peelaway;

31) In case of open tip PICCs cut the distal end to the previously defined length. Then remove at the same time, with introduce the catheter into the peel-away cannula (up to the measurement previously detected if the PICC is with a closed tip )

32) Check the blood reflux into the catheter with a suction syringe ;

33) Inject SF with the pre-connected syringe with pulsating technique;

34) Remove the introducer with the peel-away technique

35) Place sterile gauze over the point of introduction;

36) Gently remove the stylet from the catheter and cut the excess catheter length in order to assemble and connect the luerlock fitting to the catheter (if PICC with closed tip )

37) Check regular operation of suction and infusion;

38) Check the correct position of the catheter tip with guided ecg technique ;

39) Perform further washing with SF with the "stop \& go" technique and connect the needleless;

40) If necessary, cleanse the skin surrounding the insertion site with SF and sterile gauze ;

41) Dry with sterile gauze ;

42) Carefully remove the sterile adhesive drapes ;

43) Fix the catheter with sutureless system;

44) If necessary, apply a hemostatic swab to the insertion site and cover with First Aid transparent dressing;

45) Remove the remaining drapes;

46) Dispose of waste;

- Remove gloves and perform social hand washing;

- Deliver information brochures;

- $\quad$ Record in the medical record: Asepsis used;

- $\quad$ Catheter brand, caliber and lot (label

- $\quad$ adhesive);

- Site of insertion (indicate arm and vein); Length of the introduced catheter;

- Technique of introduction; Ultrasound guide;

- Any difficulties with the introduction;

- Possible hematoma or gemitium at the point of introduction; 
service costs. It is always good to avoid the use of patches or sutures, as they are not an effective alternative to ESD. Non-sterile patches can be contaminated with pathogenic bacteria, although the risk of catheter-related infections has never been exactly quantified. The use of sutures is to be avoided because it is associated with the risk of accidental needle sticks and the risk of catheter-related bacteremic infections. The integrity of the ESD should be assessed at each dressing change and the ESD should be replaced periodically according to the

manufacturer's instructions. Skin-sticking ESDs should be replaced weekly when dressing is changed. Other ESDs - such as those with subcutaneous anchoring are designed to remain in place for the entire life of the catheter, but must still be replaced if it is found that they no longer guarantee proper stabilization. The insertion site should be evaluated and disinfected with $2 \%$ chlorhexidine-based disinfectant for a certain period of time $(30 \mathrm{sec})$. At the end of the dressing change, a flush is performed, filling the lumen of the catheter with the aim of reducing the risk of intraluminal occlusion and / or catheter-related bacteremic infections. Flushing should be done using single-dose systems (e.g. single-dose vials or prefilled syringes). The pre-filled syringes available on the market appear to be effective in reducing the risk of infections and saving the time of preparing the syringe.

\section{CONCLUSION}

Although these catheters provide essential vascular access, their use poses a risk for patients of local and systemic infectious complications, i.e. local site infections, septic thrombosed phlebitis, endocarditis and other infections, including metastatic ones. The incidence of sepsis varies considerably according to the type of catheter, the frequency of manipulation and the factors associated with the operator (poor hygiene, use of incorrect aids) and patient. Although the incidence of systemic infections in the past was low, today, with the increased use of PICCs, there is an increase in the manifestation of the rate of infections that must be taken into consideration in order to prevent major episodes affecting the patient.

For this reason, the knowledge and use of useful, effective and low-cost maneuvers is required, which allow the nurse to act safely but above all not to expose the patient to infectious risks. Multiple are the strategies put in the field to the PICC and the its management but the role of the nurse that allocates a catheter is fundamental and infection prevention should be a goal to pursue.

Optimal management of the CVC / PICC ensures that no infections occur both at the site of insertion of the catheter and within the central area without incurring complications. So the nurse's aim is to protect the patient from this risk.

\section{REFERENCES}

1) aniti K., Lattyris D., Molyvdas C., Haidich A., Mouloudi E (2012) Comparison Of Oligon Catheters And Chlorhexidine-Impregnated Sponges With Standard Multilumen Central Venous Catheters For Prevention Of Associated Colonization And Infections In Intensive Care Unit Patients: A Multicenter, Randomized, Controlled Study Crit Care Medic Vol 40 N 2.

2) Association for Vascular Access. Position Statement on the Use of Real-Time Imaging Modalities for Placement of Central Venous Access Devices. 2008.

3) Baskin JL, Pui CH, Reiss U et al. Management of occlusion and thrombosis associated with long-term indwelling central venous catheters. Lancet 2009 Jul 11; 374 (9684): 159-69

4) Burns DF. Developing a successful radiology nursing peripherally inserted central catheters and midline insertion program. J Radiol Nurs. 2006; 25 (4): 116-18.

5) Canzan F in Battaglia E, Vanzetta M, Tolomeo S. Infusion Therapy - Tools and methods. Italy: McGraw-Hill (1st ed), 2011,pg. 9-13.

6) Cheung E, Baerlocher MO, Asch M, Myers A. Venous access. A practical review for 2009. Can Fam Physician. 2009; 55 (5): 494-6.

7) Freytes CO Thromboembolic complications related to central venous access catheters in cancer patients. Seminars in thrombosis and hemostasis 2007 Jun; 33 (4): 389-96

8) Garland J., Colleen P., Mneller C., Otten D., Naples M., Pellegrini J (2001) Gluconate-Impregnated Dressing For Prevention Of Central Venous Catheter A Randomized Trial Comparing Povidone-Iodine To A Chlorhexidine Pediatrics, Vol 11 N 13 Pages 90-101.

9) Guyatt GH, Akl EA, Crowther M et al. Executive summary: Antithrombotic Therapy and Prevention of Thrombosis, 9th ed: American College of Chest Physicians Evidence-Based Clinical Practice Guidelines. Chest 2012 Feb; 141 (2 Suppl): $7 S-47 S$

10) Hamilton HC, Foxcroft DR. Central venous access sites for the prevention of venous thrombosis, stenosis and infection in patients requiring long-term intravenous therapy 2007.

11) Hill M., Balwin L., Slaughter Jc., Walsh Wf, Witkamp H (2010) A Silver-Alginate-Coated Dressing To Reduce Peripherally Inserted Central Catheter (Picc) Infections In Nicu Patients: A Pilot Randomized Controlled Trial Journal Of Perinatology (2010) 30, 469-473

12) Infusion Nurses Society [INS]. Infusion nursing standards of practice: Site selection and device placement. J Infus Nurs. 2006; 29 (1s): s37-s39. 
13) Kearon C, Kahn SR, Agnelli G et al. Antithrombotic therapy for venous thromboembolic disease: American College of Chest Physicians Evidence-Based Clinical Practice Guidelines (8th Edition). Chest 2008 Jun; 133 (6 Suppl): $454 S-545 S$ (full text)

14) Kucher N Clinical practice. Deep-vein thrombosis of the upper extremities. The New England journal of medicine 2011 Mar 3; 364 (9): 861-9

15) Levy I., Katz J., Solter E., Samra Z., Vidne B (2005) Chlorhexidine-Impregnated Dressing For Prevention Of Colonization Of Central Venous Catheters In Infants And Children A Randomized Controlled Study Pediatr Infect Dis $J$ 2005; 24: 676 - 679

16) Maddukuri P, Downey BC, Blander JA, Pandian NG, Patel AR. Echocardiographic diagnosis of air embolism associated with central venous catheter placement: case report and review of the literature. Echocardiography 2006 Apr; 23 (4): 315-8.

17) Milling TJ Jr, Rose J, Briggs WM, Birkhahn R, Gaeta TJ, Bove JJ. Randomized, controlled clinical trial of point-ofcare limited ultrasonography assistance of central venous cannulation: the third Sonography Outcomes Assessment Program (SOAP- 3) Trial. Crit Care Med. 2005; 33 (8): 1764-9.

18) Pittiruti M, Hamilton H, Biffi R et al. ESPEN Guidelines on Parenteral Nutrition: central venous catheters (access, care, diagnosis and therapy of complications). Clinical nutrition (Edinburgh, Scotland) 2009 Aug; 28 (4): 365-77

19) Prandoni P Prophylaxis of catheter-related thrombosis in cancer patients. Lancet 2009 Feb 14; 373 (9663): 523-4

20) Safdar N., Maki D (2005) Risk Of Catheter-Related Bloodstream Infection With Peripherally Inserted Central Venous Catheters Used In Hospitalized Patients Chest, V 128 N 2, Pag 489-494

21) Shokoohi H, Boniface K, McCarthy M, Al-Tiae TK, Sattarian M, Ding R, Liu YT, Pourmand A, Schoenfeld E, Scott J, Shesser R, Yadav K. Ultrasound-guided peripheral intravenous access program is associated with a marked reduction in central venous catheter use in noncritically ill emergency department patients. Annals of Emergency Medicine. 2013; 61 (2): 198-203.

22) Supplement to the January / February 2016 issue Volume 39, Issue ISISSN 1533-1458 www.journalofinfusionnursing. com ; Journal of Infusions on Nursing 